\section{Prevalencia de caries de la infancia temprana y factores de riesgo asociados}

\section{RESUMEN}

Antecedentes: la caries de la infancia temprana es una enfermedad bacteriana que se presenta en niños menores de 71 meses de edad, con una prevalencia que puede llegar hasta $90 \%$ en poblaciones altamente vulnerables.

Objetivo: determinar la prevalencia de caries y los factores de riesgo en pacientes menores de 48 meses, así como la relación existente entre los factores de riesgo y las caries.

Materiales y métodos: estudio descriptivo y transversal en el que se incluyeron 63 niños sin distinción de género, desde los 9 hasta los 48 meses de edad (edad promedio de 26.8 meses). Se utilizó un cuestionario en el que se registraron factores de riesgo para caries. Se efectuó examinación clínica registrando la higiene oral con el índice de O'Leary y la prevalencia de caries dental mediante el índice ceo-d (para dientes temporales) propuesto por la OMS, con la modificación del componente cariado utilizando la escala de Pitts y Fyffe.

Resultados: la prevalencia de caries fue de $35 \%$, el ceo-d promedio fue de 1.0 ( \pm 1.86$)$. La lesión de mancha blanca estuvo presente en $73 \%$ de los casos de caries. No se encontró asociación significativa entre la prevalencia de las caries y la frecuencia o calidad de la higiene oral, cariogenicidad de la dieta o el uso de fluoruros como método preventivo de caries. El análisis se realizó utilizando medidas de tendencia central y la prueba de $\chi^{2}$.

Conclusión: debido a la alta prevalencia de manchas blancas es necesaria la creación de programas preventivos que eduquen a las madres o cuidadores sobre los factores de riesgo de caries, fomentando el autocuidado como estrategia de prevención.

Palabras claves: caries dental, infancia temprana, prevención primaria.

\section{Prevalence of early childhood caries and associated risk factors}

\section{ABSTRACT}

Background: Early childhood caries is a bacterial disease that occurs in children under 71 months of age, with a prevalence of up to 90 in highly vulnerable populations.
Fernando Javier Aguilar-Ayala ${ }^{1}$ Claudia Gabriela Duarte-Escobedo 2 Marina Eduviges Rejón-Peraza ${ }^{1}$ Rodrigo Serrano-Piña ${ }^{1}$ Alicia Leonor Pinzón-Te ${ }^{1}$

1 Profesor de la Maestría en Odontología Infantil, Facultad de Odontología, Universidad Autónoma de Yucatán.

2 Exresidente de la Maestría en Odontología Infantil, Facultad de Odontología, Universidad Autónoma de Yucatán.
Recibido: 13 de diciembre 2013

Aceptado: 16 de mayo 2014

Correspondencia: Dr. Fernando Javier Aguilar Ayala

Calle 59-A \#527 x76 y 78 Centro

CP. 97000 Mérida, Yucatán

Cel. 9991282205, (999)9236752 Ext.110

faguilar@uady.mx

Este artículo debe citarse como

Aguilar-Ayala FJ, Duarte-Escobedo CG, Rejón-Peraza ME, Serrano-Piña R, Pinzón-Te AL. Prevalencia de caries de la infancia temprana y factores de riesgo asociados. Acta Pediat Mex 2014;35:259-266. 
Objective: Evaluation of the prevalence of caries and its risk factors in patients under 48 months of age and the relationship between risk factors and caries.

Materials and Methods: descriptive and cross-sectional study which included 63 children, without distinction of gender, between 9 and 48 months of age (average 26.8 months). We used a questionnaire in which risk factors were annotated; clinical examination was performed, recording oral hygiene by the $\mathrm{O}^{\prime}$ Leary index and the prevalence of the dental caries with def-t proposed by $\mathrm{WHO}$, with the modification of the decay component using the Pitts and Fyffe scale.

Results: Caries prevalence was 35\%, the def-t mean was $1.0( \pm 1.86)$. The white spot lesion was present in $73 \%$ of caries cases. No significant association was observed between caries prevalence and frequency or quality of oral hygiene, cariogenicity of the diet, or the use of fluoride in caries prevention. Data was analyzed by means using the chi-square test.

Conclusion: due to the high prevalence of white spots, is necessary to create prevention programs that educate mothers or caregivers about the caries risk factors and its control, promoting self-care as a prevention strategy.

Key words: dental caries, early childhood, primary prevention.

\section{ANTECEDENTES}

Los niños en edades tempranas son altamente vulnerables a problemas de salud en general. Muchas veces implican problemas de salud bucal como la caries dental considerada como una enfermedad transmisible de origen multifactorial, plenamente relacionada con higiene oral defectuosa, alimentación nocturna, alto consumo de azucares, colonización bacteriana y bajo nivel socioeconómico de los padres. Ésta, puede presentar graves repercusiones como dolor intenso, infecciones faciales, hospitalizaciones y visitas a urgencias, así como disminución en el desarrollo físico del infante, ${ }^{1}$ alto costo de tratamiento y disminución en la calidad de vida. ${ }^{2}$

La Academia Americana de Odontología Pediátrica (AAPD) define la caries de la infancia temprana como la presencia de uno o más órganos dentarios con caries, perdidos u obturados, en niños de 71 meses de edad o menores. Cuando esta condición no es tratada puede llegar a afectar a todos los dientes presentes en la cavidad bucal, lo que se denomina caries rampante. ${ }^{3}$

La caries de la infancia temprana, antes conocida como caries de biberón, es una forma particularmente virulenta de caries que inicia poco después de la erupción dental y se desarrolla en las superficies dentales lisas con un rápido progreso. Suele mostrar un patrón característico: caries en los incisivos superiores, los molares de ambas arcadas pero no en los incisivos inferiores; este patrón se relaciona con la secuencia de erupción y la posición de la lengua durante la alimentación. ${ }^{1}$ La caries de la infancia temprana está asociada con la ingesta excesiva de cualquier líquido azucarado como la leche, fórmulas, jugos de frutas, refrescos, la alimentación a libre 
demanda del seno materno y la falta de higiene después de la ingesta. ${ }^{4-11}$

Los factores de riesgo como la dieta, la higiene oral, la exposición a fluoruros y el uso prolongado de biberón, entre otros, son los principales causantes de caries de la infancia temprana. ${ }^{6}$ Estos son atributos que le confieren cierto grado de susceptibilidad a los pacientes infantiles, constituyendo una probabilidad medible, con valor predictivo, y que al modificarse aportan ventajas para la prevención individual, grupal o comunitaria.

La prevalencia de la caries de la infancia temprana varía de $3.1 \%$ a $90 \%$ dependiendo de la vulnerabilidad de las poblaciones, sobre todo cuando éstas pertenecen a grupos de nivel socioeconómico bajo. ${ }^{12}$ Más datos epidemiológicos nos indican que el abordaje efectivo, para el control de la caries de la infancia temprana, debe estar basado en la prevención primaria. ${ }^{13}$

\section{MATERIALES Y MÉTODOS}

Se estudiaron 63 niños de entre 9 y 48 meses de edad, sin distinción de género si cumplían el único criterio de inclusión que fue estar inscrito en el programa de Estimulación Temprana de la Unidad Universitaria de Inserción Social de la Universidad Autónoma de Yucatán; lugar donde se llevó a cabo el estudio durante los meses de noviembre y diciembre de 2011. Antes del estudio los padres o tutores firmaron un consentimiento informado y respondieron un cuestionario diseñado para registrar los datos personales de los niños y niñas incluidos y para medir algunos de los factores de riesgo para la caries de la infancia temprana.

Las variables incluidas en el estudio fueron la prevalencia de caries dental mediante el índice ceo-d modificado, el índice de placa dentobacteriana de $\mathrm{O}^{\prime}$ Leary $^{14}$ y algunos factores de riesgo como la frecuencia de la higiene oral, el amamantamiento o uso nocturno del biberón, la higiene oral previa al sueño, la cariogenicidad de la dieta y el uso de fluoruros tópicos.

En el estudio se tomaron en cuenta el género, la edad (dividida en 3 rangos), la cantidad de higiene oral de acuerdo con: 1) si se realizaba cepillado dental de 0 a 1 vez al día o 2 o más veces al día; 2) de acuerdo con el porcentaje encontrado con el índice de higiene oral de O'Leary, aceptable, cuestionable y deficiente. El tipo de dieta fue clasificada como no cariogénica cuando se ingerían menos de 4 porciones de carbohidratos diarios y como cariogénica si se ingerían más de 5 porciones de carbohidratos diarios, registrados en el recordatorio de las 24 horas previas. También se aplicó una encuesta sobre ingesta de alimentos cariogénicos: una puntuación entre 10 y 33 se consideró de baja cariogenicidad; por arriba de 34 se consideró cariogenicidad moderada-alta. ${ }^{11}$ Asimismo, para evaluar la exposición a fluoruros se tomó en cuenta el uso de pasta dental durante el cepillado dental y si el paciente había recibido previamente aplicación tópica de flúor.

Un único investigador llevó a cabo la examinación clínica de los niños, en la posición rodilla con rodilla con la ayuda de una lámpara frontal, espejo dental no. 5 y el uso de los medios de protección que indica la Norma Oficial Mexicana para la prevención de enfermedades bucales.

La placa dentobacteriana ${ }^{15}$ fue teñida con solución reveladora y registrada con los criterios del índice de higiene oral de O'Leary, en el cual son registradas las superficies dentales teñidas con solución reveladora de los órganos dentales presentes en la boca. Para calcular el índice individual se toma en cuenta la siguiente ecuación:

$$
\begin{aligned}
& \begin{array}{c}
\text { Cantidad de } \\
\text { superficies } \\
\text { teñidas } \times 100 \\
\begin{array}{c}
\text { Total de superficies } \\
\text { presentes }
\end{array}
\end{array}=\begin{array}{l}
\% \text { (Índice de higiene } \\
\text { oral de O' Leary) }
\end{array} \\
&
\end{aligned}
$$


Se tomaron en cuenta los parámetros, O'Leary (cuadro 1) en cuanto al porcentaje obtenido del índice de placa dentobacteriana. ${ }^{14}$

Cuadro 1. Parámetros del índice O'Leary de acuerdo con el porcentaje de placa dentobacteriana

\begin{tabular}{lc}
\hline Condición & Parámetro \\
Aceptable & $0-12 \%$ \\
Cuestionable & $13-23 \%$ \\
Deficiente & $24-100 \%$.
\end{tabular}

Posterior a la detección y, para realizar el examen bucal, la placa dentobacteriana fue removida con gasa, previamente humedecida con agua purificada, registrándose en un odontograma la experiencia de caries de acuerdo con el índice ceo-d propuesto por la OMS, con la modificación del componente cariado utilizando la escala de Pitts y Fyffe que toman los siguientes criterios:

Caries inicial: clínicamente no se detecta pérdida de sustancia. Las fosas y fisuras presentan cambio de coloración; se aprecian áreas opacas o manchas blancas con pérdida de brillo en las superficies lisas (como de tiza blanca).

Caries de esmalte: clínicamente hay pérdida de sustancia en fosas, fisuras y superficies lisas. La textura de la cavidad puede parecer desmoronable o arenosa, sin evidencia de estar afectando la dentina.

Caries de dentina: cavidad franca que afecta a la dentina, las paredes de la lesión pueden estar reblandecidas.

Caries con afección pulpar: cavidad profunda con probable afección pulpar. ${ }^{16}$

Para efecto de la investigación del índice ceo-d se consideraron los siguientes códigos:
A: sano

B: cariado

B1: caries inicial

B2: caries de esmalte

B3: caries de dentina

B4: caries con afección pulpar

C: obturado con caries

D: obturado sin caries

E: indicados para extracción por caries

El análisis estadístico consistió primeramente en describir, mediante porcentajes y frecuencias, los grupo de edades de los niños, género y si habían visto o no al dentista. Posteriormente se obtuvieron los puntajes promedios y sus desviaciones estándar para las variables: índice ceo-d, índice de O'Leary y edad. Se clasificó el índice de O'Leary para todos los dientes de acuerdo con el parámetro establecido a priori.

Además, se obtuvo la prevalencia de niños con caries y las frecuencias y porcentajes de los dientes con lesiones no cavitadas, lesiones de esmalte y lesiones de dentina. También se obtuvieron los índices de prevalencia para cada uno de los factores de riesgo.

Por último, se realizó la prueba $\chi^{2}$ de independencia, con un nivel de confianza de $95 \%$, para determinar la relación entre la caries y cada uno de los factores de riesgo, el género o la edad. Después del estudio todos los niños, junto con sus cuidadores, fueron sometidos a un programa de salud bucodental específico durante 6 meses recibiendo información sobre caries y su prevención.

\section{RESULTADOS}

El estudio incluyó 63 niños de 9 a 48 meses de edad (media $=26.87 \pm 11.65$ ), divididos en tres grupos de edades: 9 a 20 meses $(31.75 \%$; 20 niños), de 21 a 33 meses (34.92\%; 22 niños) y de 34 a 48 meses (33.33\%; 21 niños). Se exami- 
naron 35 niñas $(55.56 \%)$ y 28 niños $(44.44 \%)$. La mayoría $(66.67 \% ; 42)$ de los niños evaluados nunca había visitado al dentista. En la población estudiada se encontró mayor frecuencia de caries a partir de los 2 años de edad, con un 53.13\% de los casos.

De todos los evaluados $12.96 \%$ (7) seguía siendo amamantado, mientras que $77.78 \%$ (49) continuaba siendo alimentado a través de biberón.

Tenía dieta cariogénica durante el día 74.6\% (47) de los pacientes. El $100 \%$ de los pacientes era amamantado o alimentado con biberón antes de dormir y, de éstos, únicamente el 34.92\% (22) tenía higiene dental antes del sueño. En $87.3 \%$ de los casos la higiene oral era realizada máximo 1 vez al día. El cepillo dental fue utilizado en $84.1 \%$ de los niños. En cuanto a la aplicación de fluoruro tópico únicamente $25.4 \%$ lo había utilizado (cuadro 2).

En cuanto a la valoración de la placa dentobacteriana de las 3716 superficies dentales examinadas en 757 había placa para un promedio de $20 \%$ de superficies dentales afectadas lo cual, según los parámetros de O'Leary, representa una higiene oral cuestionable.
La prevalencia de caries fue de $34.92 \%$ (22) y el promedio ceo-d (dientes temporales) fue 1.00 ( \pm 1.86$)$. En los niños con caries $73 \%$ de las lesiones fueron no cavitadas, $21 \%$ afectaba únicamente al esmalte y en $6 \%$ de los casos se afectaba la dentina. No se observaron restauraciones ni casos en los que estuviera indicada extracción dental.

La asociación entre caries y género sexual no fue estadísticamente significativa; sin embargo, la asociación entre los grupos de edad y la presencia de caries sí fue estadísticamente significativa $(p<0.05)$. De los niños entre 9 y 20 meses de edad $80 \%$ se encontraba libres de caries, mientras que $61.9 \%$ de los de que tenían de 34 a 48 meses presentó caries. No se observó influencia de la higiene oral, la dieta o el uso de fluoruros dentales sobre la prevalencia de caries $(p>0.05)$ (cuadro 3).

\section{DISCUSIÓN}

Estudios como el de Gridefjord (Suecia) reportan una prevalencia de caries de la infancia temprana de $11.4 \% ;{ }^{17}$ el de King (China) infiere 18\% de prevalencia ${ }^{18}$ y contrasta con un aumento por lo observado en este estudio (34.92\%). Sin embargo, este dato es superado en estudios similares de

Cuadro 2. Frecuencia y porcentaje de factores de riesgo

\begin{tabular}{|c|c|c|}
\hline Factor & & \\
\hline \multirow[t]{2}{*}{ Higiene oral } & Sí & No \\
\hline & $88.7 \%(54)$ & $14.3 \%(9)$ \\
\hline \multirow[t]{2}{*}{ Frecuencia de la higiene oral } & 0-1 vez & 2 o más veces \\
\hline & $87.3 \%(55)$ & $12.7 \%(8)$ \\
\hline \multirow[t]{2}{*}{ Amamantamiento/biberón nocturno } & Sí & No \\
\hline & $100 \%(63)$ & 0 \\
\hline \multirow[t]{2}{*}{ Higiene oral después del amamantamiento/biberón nocturno } & Sí & No \\
\hline & $34.9 \%(22)$ & $65.1 \%(41)$ \\
\hline \multirow[t]{2}{*}{ Dieta cariogénica ${ }^{26}$} & Sí & No \\
\hline & $74.6 \%(47)$ & $25.4 \%(16)$ \\
\hline \multirow[t]{2}{*}{ Potencial cariogénico de la dieta } & Bajo & Medio-alto \\
\hline & $20.6 \%(13)$ & $79.4 \%(50)$ \\
\hline \multirow{2}{*}{ Uso de fluoruros tópicos } & Sí & No \\
\hline & $25.4 \%(16)$ & $74.6 \%(47)$ \\
\hline
\end{tabular}


Cuadro 3. Porcentaje y frecuencia de caries dental

\begin{tabular}{|c|c|c|c|c|c|c|}
\hline & Variables & Caries & Libre de caries & $\chi^{2}$ & GL** & $p$ \\
\hline \multirow[t]{2}{*}{ Sexo } & Femenino & $54.55 \%(12)$ & $56.10 \%(23)$ & .014 & 1 & 0.9059 \\
\hline & Masculino & $44.45 \%(10)$ & $43.90 \%(18)$ & & & \\
\hline \multirow[t]{3}{*}{ Edad } & 9 a 20 meses & $20 \%(4)$ & $80 \%(16)$ & 10.127 & 2 & $0.0063^{*}$ \\
\hline & 21 a 33 meses & $22.7 \%(5)$ & $77.3 \%(17)$ & & & \\
\hline & 34 a 48 meses & $61.9 \%(13)$ & $38.1 \%(8)$ & & & \\
\hline \multirow{5}{*}{$\begin{array}{c}\text { Higiene } \\
\text { oral }\end{array}$} & $0-1$ vez & $38.2 \%(21)$ & $61.8 \%(34)$ & 2.027 & 1 & 0.1545 \\
\hline & 2 o más veces & $12.5 \%(1)$ & $87.5 \%(7)$ & & & \\
\hline & Aceptable (0-12\% PDB**) & $9.1 \%(1)$ & $90.9 \%(10)$ & 4.320 & 2 & 0.1153 \\
\hline & Cuestionable (13-23\% PDB) & $36 \%(9)$ & $64 \%(16)$ & & & \\
\hline & Deficiente (24-100\% PDB) & $44.4 \%(13)$ & $55.6 \%(15)$ & & & \\
\hline \multirow[t]{4}{*}{ Dieta } & Cariogénica & $36.2 \%(17)$ & $63.8 \%(30)$ & 0.127 & 1 & 0.7214 \\
\hline & No cariogénica & $31.3 \%(5)$ & $68.8 \%(11)$ & & & \\
\hline & Baja cariogenicidad & $23.1 \%(3)$ & $76.9 \%(10)$ & 1.011 & 1 & 0.3147 \\
\hline & Mediana-alta cariogenicidad & $38 \%(19)$ & $62 \%(31)$ & & & \\
\hline \multirow{4}{*}{$\begin{array}{l}\text { Uso de } \\
\text { fluoruro }\end{array}$} & En pasta dental & $40.5 \%(17)$ & $59.5 \%(25)$ & 1.711 & 1 & 0.1908 \\
\hline & No usan pasta dental & $23.8 \%(5)$ & $76.2 \%(16)$ & & & \\
\hline & Aplicación tópica & $37.5 \%(6)$ & $62.5 \%(10)$ & 0.063 & 1 & 0.8021 \\
\hline & No se le ha aplicado tópicamente & $66 \%(31)$ & $34 \%(16)$ & & & \\
\hline
\end{tabular}

*Se encontró asociación entre el factor y la caries. ${ }^{* *} \mathrm{GL}$ : grados de libertad; PDB: placa dentobacteriana.

otros países encontrando prevalencias de $41.4 \%$ en Brasil, ${ }^{19} 53 \%$ en Canadá, ${ }^{20} 63.17 \%$ en India, ${ }^{21}$ $74.9 \%$ en Colombia ${ }^{22}$ hasta $90 \%$ en Filipinas, en niños de hasta 4 años de edad. ${ }^{23}$

En nuestro estudio la prevalencia de caries y el ceo-d promedio fueron, respectivamente, $35 \%$ y 1.00 ( \pm 1.8). Así, la prevalencia encontrada por nosotros fue menor a la encontrada en otro estudio realizado en México por Montero $(59.5 \%){ }^{5}$

Uno de los factores de riesgo a caries con mayor presencia en nuestro estudio fue el consumo de dieta cariogénica, presente en $74.6 \%$ de los pacientes, aún cuando no se encontró que se asociara de manera estadísticamente significativa con la caries, similar a lo reportado por Santos. ${ }^{19}$ Es necesario el control de hábitos dietéticos mediante la reducción de ingesta alta de carbohidratos fermentables a lo largo del día, debido a que esto se convierte en un factor de riesgo para la implantación y colonización de bacterias y, por ende, para la desmineralización del tejido dentario.

Dentro de los carbohidratos, la sacarosa es la de mayor capacidad cariogénica y su introducción en la dieta moderna está relacionada con la alta prevalencia de caries, especialmente en las superficies lisas de los dientes. ${ }^{19}$

Otro estudio tampoco encontró asociación estadísticamente significativa entre la placa dentobacteriana y la caries; sin embargo, en otros casos se ha encontrado que la presencia de placa dentobacteriana es el factor más fuertemente asociado con la caries. ${ }^{5,24}$ En la población en estudio se encontró asociación directamente 
proporcional entre grupos de edad y caries; se observó que a mayor edad mayor prevalencia de caries, tal como se reporta otros estudios. ${ }^{19,24}$

La lesión de caries dental más frecuentemente encontrada en este estudio fue la mancha blanca (73\% de los casos), coincidente con lo que se reporta en otros estudios. ${ }^{19,24,25}$ Estas lesiones se pueden desarrollar incluso en niños menores de 12 meses de edad, por lo cual es recomendable que la primera visita al odontopediatra se efectúe antes de esta edad, para que el especialista en el área de infantil, o bien el odontólogo de práctica general, pueda identificar y controlar los factores de riesgo a los cuales está expuesto el niño y evitar el deterioro de los órganos dentarios por el avance de la enfermedad.

Los resultados de este estudio revelan claramente la necesidad de crear mejores programas de prevención que sirvan de guía para padres y cuidadores. Dichos programas deberían estar enfocados en cómo llevar a cabo una higiene oral efectiva en los niños, educarse y educar paulatinamente en la creación de buenos hábitos de alimentación, desde los primeros años de vida. No menos importante es proveer información relacionada con el cuidado dental desde el embarazo, las citas al odontólogo para dar seguimiento a la salud y los cuidados dentales para el bebé, ya que es más fácil prevenir hábitos inadecuados que tratar de eliminarlos después de que se han instalado en el día a día.

La prevención de caries de la infancia temprana depende de esfuerzos multidisciplinarios, que incluyen a diferentes profesionales de la salud y al gobierno como responsable de invertir en salud.

\section{CONCLUSIONES}

La prevalencia de caries en este estudio fue de $35 \%$ (y la media del ceo-d fue $1.0[ \pm 1.86]$ ), resultado que consideramos como moderado aún cuando aumenta con la edad manifestándose, en términos generales, como mancha blanca. No menos importante es considerar la posibilidad de que por la falta de conocimiento sobre salud bucal se sigan presentando, a edades tan tempranas, factores de riesgo para caries: baja frecuencia en la higiene bucal, amamantamiento o alimentación con biberón nocturno, falta de higiene bucal durante la noche, alimentación con una dieta cariogénica con riesgo medioalto y bajo uso de flúor dental, entre otros. Por lo mencionado anteriormente se percibe la necesidad de implementar programas preventivos que eduquen a las madres o cuidadores acerca de los factores de riesgo para caries y de su control, fomentando con ello el autocuidado como estrategia de prevención.

\section{REFERENCIAS}

1. Alonso-Noriega MJ, Karakowsky L. Caries de la infancia temprana. Perinatología y Reproducción Humana 2009;23(2):90-7.

2. Palma C, Cahuana A, Gómez L. Guía de orientación para la salud bucal en los primeros años de vida. Acta Pediátrica Española 2010;68(7):351-7.

3. Seminario A, Ivancaková R. Early childhood caries. Acta Médica (Hradec Králové) 2003;46(3):91-4.

4. López del Valle L, Velázquez-Quintana $Y$, Weinstein $P$, et al. Early childhood caries and risk factors in rular Puerto Rican children. ASDC J Dent Child 1998;65(2):132-5.

5. Montero-Canseco D, López-Morales P, Castrejón-Pérez R. Prevalencia de caries de la infancia temprana y nivel socioeconómico familiar. Revista Odontológica de México 2011;15(2):96-102.

6. Menchaca-Montellano G, Gil-Orduña C, San Martín-Brieke W, Ochoa-Cáceres S, Cabrera-Serrano S. Factores de riesgo en pacientes de caries temprana de la infancia del Departamento de Estomatología del Hospital para el Niño Poblano. Oral 2004;16:230-2.

7. Cárdenas-Jaramillo D. Odontología Pediátrica. Medellín, Colombia: Corporación para Investigaciones Biológicas, 2009; pp. 117-20.

8. Rodríguez-Vilchis $L$, Contreras-Bulnes R, Arjona-Serrano $M$, Soto-Mendieta R, Alanis-Tavira J. Prevalencia de caries y conocimientos sobre salud-enfermedad bucal de niños (3 a 12 años) en el Estado de México. Revista ADM 2006;63(5):170-5.

9. Juárez-López L, Murrieta-Pruneda F, Ortiz-Cruz E. Prevalencia de caries y su asociación con el estado nutri- 
cional y hábitos higiénicos en preescolares. Rev AMOP 2006;18(2):28-32.

10. Romero-Balza J, Juárez-López M. Prevalencia y factores de riesgo de la caries dental en escolares de Ciudad Nezahualcóyotl. Medicina Oral 2006;8(4):163-7.

11. Vaisman B, Martínez M. Asesoramiento dietético para el control de caries en niños. Revista Latinoamericana de Ortodoncia y odontopediatría 2004;1-11.

12. Yiu C, Wei S. Management of rampant caries in children. Quitessence International 1992;23(3):159-68.

13. Arango $M$, Baena $G$. Caries de la infancia temprana y factores de riesgo. Revisión de la literatura. Revista Estomatología 2004;12(1):59-65.

14. Newman M, Takei H, Carranza F. Periodontología Clínica. 9a edición. Ciudad de México: McGraw-Hill; 2007; pp. 709.

15. Alaluusua $\mathrm{S}$, Malmivirta R. Early plaque accumulation - a sign for caries risk in young children. Community Dentistry and Oral Epidemiology 1994;22(5):273-6.

16. Palma C, Cahuana A. Pautas para la Salud Bucal en los primeros años de vida. Odontol Pediatr 2010;9(2):179-89.

17. Gridefjord M, Dahllöf G, Ekström G, Höjer B, Modéer T. Caries prevalence in 2.5 years-old children. Caries Res 1993;27:505-10.

18. King NM, Wu II, Tsai JS. Caries prevalence and distribution, and oral health habits of zero- to four years old children in Macau, China. J Dent Child 2003;70(3):243-9.
19. Santos A, Mendes V. Caries prevalence and risk factor among children aged 0 to 36 months. Pesqui Odontol Bras 2001;3(16):203-8.

20. Schroth R, Dahl P, Haque M, Kliewer E. Early childhood caries among Hutterite preschool children in Manitoba, Canada. Rural Remote Health 2010;10(4):1535.

21. Sankeshwari RM, Ankola AV, Tangade PS, Hebbal MI. Feeding Habits and Oral Hygiene Practices as Determinants of Early Childhood Caries in 3 to 5 year old Children of Belgaum City, India. Oral Health \& Preventive Dentistry 2012;10(3):283-290.

22. Saldarriaga-Cadavid $A$, Arango-Lince $M$, Cossio-Jaramillo $M$. Dental caries in the primary dentition of a Colombian population according to the ICDAS criteria. Braz Oral Res 2010;24(2):211-6.

23. Cariño KM, Shinada K, Kawaguchi Y. Early childhood caries in northern Philippines. Community Dent Oral Epidemiol 2003;31:81-9.

24. Warren J, Levy S, Kanellis M. Dental Caries in the Primary Dentition: Assessing Prevalence of Cavitated and Noncavitated Lesions. Journal of Public Health Dentistry 2002;62(2):109-114.

25. Rodríguez-Llanes R, Traviesas-Herrera EM, LavanderaCarballido E, Duque- Hernández M. Factores de riesgo asociados con la caries dental en niños de círculos infantiles. Revista Cubana de Estomatología 2009;46(2):0-0. 